RECEYYD

FER 201997

OSTI

PVM and IP Multicast
Thomas H. Dunigan

Kara A. Hall
MASTEF. DISTRIBUTON OF THIS DOCUMENT IS UNLW 
This report has been reproduced directly from the best available copy.

Available to DOE and DOE contractors from the Office of Scientific and Technical Information, P.O.Box 62, Oak Ridge, TN 37831; prices available from (423) 576-8401, FTS 626-8401.

Available to the public from the National Technical Information Service, U.S. Department of Commerce, 5285 Port Royal Rd., Springtield, VA 22161

This report was prepared a an account of work sponsored by an agency of the United States Government. Neither the United States Government nor any agency thereof, nor any of their employees, makes any warranty, express or implied, or assumes any legal liability or responsibility for the accuracy, compleleness, or usefulness of any information, apparatus, product, or process disclosed, or represents that its use would not infringe privately owned rights. Reference herein to any specific commercial product, process, or service by trade name, trademark, manufacturer, or otherwise, does not necessarily constitute or imply its endorsement, recommendation, or favoring by the United States Government or any agency thereof. The view and opinions of authors expressed herein do nol necessarily state or reflect those of the UnitedStalesGovernment or any agency thereot. 


\section{DISCLAIMER}

Portions of this document may be illegible electronic image products. Images are produced from the best available original document. 
Computer Science and Mathematics Division

Mathematical Sciences Section

\section{PVM AND IP MULTICAST}

Thomas H. Dunigan and Kara A. Hall

Mathematical Sciences Section

Oak Ridge National Laboratory

P.O. Box 2008, Bldg. 6012

Oak Ridge, TN 37831-6367

thd@ornl.gov hall@cs.utk.edu

Date Published: December 1996

Research was supported by the Applied Mathematical Sciences Research Program of the Office of Energy Research, U.S. Department of Energy.

Prepared by the

Oak Ridge National Laboratory

Oak Ridge, Tennessee 37831

managed by

Lockheed Martin Energy Research Corp.

for the

U.S. DEPARTMENT OF ENERGY

under Contract No. DE-AC05-96OR22464 


\section{Contents}

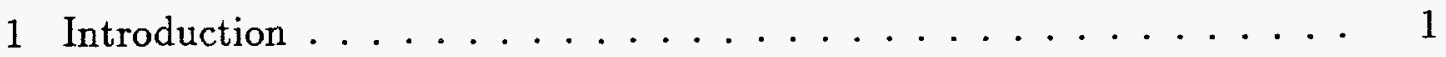

2 Multicast ..................... 1

2.1 Multicast and Broadcast on LANs and Multiprocessors . . . . . 2

2.2 Internet Protocol (IP) Multicast . . . . . . . . . . . 2

2.3 Reliable Multicast . . . . . . . . . . . . . . 4

3 Reliable IP multicast in PVM. . . . . . . . . . . . 6

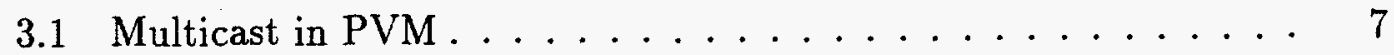

3.1 .1 PVM message-passing . . . . . . . . . . 7

3.1 .2 PVM's pvm_mcast() protocol . . . . . . . . . 9

3.1 .3 PVM multicast address . . . . . . . . . . . 9

3.1 .4 Initiating multicast $\ldots \ldots \ldots \ldots \ldots \ldots$

3.1 .5 Sending multicast . . . . . . . . . . . 11

3.1 .6 PVM Multicast routing . . . . . . . . . . . . . 11

3.2 Adding IP multicast to PVM . . . . . . . . . . . . . 12

3.2 .1 The pvm.jpmcast() protocol . . . . . . . . . 12

3.2 .2 IP multicast socket . . . . . . . . . . . . 12

3.2 .3 Initiating IP multicast $\ldots \ldots \ldots \ldots \ldots \ldots \ldots$

3.2 .4 Sending IP multicast $\ldots \ldots \ldots \ldots \ldots$

3.2.5 Acknowledging IP multicast . . . . . . . . . . . . 14

3.3 PVM IP multicast Performance . . . . . . . . . . . . 16

3.3.1 LAN Performance of pvm_ipmcast() . . . . . . . . 16

3.3.2 WAN Performance of pvm_ipmcast() . . . . . . . . 17

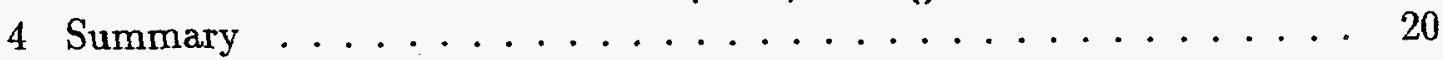

4.1 Limitations . . . . . . . . . . . . . . . . . 21

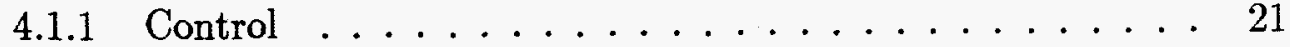

4.1 .2 Availability . . . . . . . . . . . . . . . 22

4.1.3 Address and Port assignments . . . . . . . . . . . . . 22

4.1.4 Bandwidth and Routing . . . . . . . . . . . 23

4.2 Future Work . . . . . . . . . . . . . . . . . 23

5 References . . . . . . . . . . . . . . . 24 


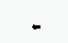




\title{
PVM AND IP MULTICAST
}

\author{
Thomas H. Dunigan and Kara A. Hall
}

\begin{abstract}
This report describes a 1994 demonstration implementation of PVM that uses IP multicast. PVM's one-to-many unicast implementation of its pvm_mcast() function is replaced with reliable IP multicast. Performance of PVM using IP multicast over local and wide-area networks is measured and compared with the original unicast implementation. Current limitations of IP multicast are noted.
\end{abstract}




\section{Introduction}

PVM, Parallel Virtual Machine [7], is a message-passing system that allows a collection of heterogeneous computers on a network to function as a virtual parallel computer. PVM supplies the functions to automatically start up tasks on the logical distributed-memory computer and allows the tasks to communicate and synchronize with each other. PVM is used by researchers to speed up computing applications by harnessing the power of workstations and supercomputers connected by a network. This report describes describes extensions made to PVM to support IP multicast as part of PVM's one-to-many communications protocol.

This report is a subset of a larger research effort by Hall [9] that looked at many of the research issues in reliable multicast protocols. Research issues studied in adding IP multicast support to PVM include:

- multicast reliability

- delivery and response semantics

- group structure

- reliable multicast methodologies

- performance implications

This report summarizes the choices made within these research issues. The following section describes multicast protocols. Section 3 describes the use of multicast in PVM, the extensions made to support IP multicast, and the effects on performance. The final section summarizes the current (1994) limitations of IP multicast in PVM and IP multicast on the Internet.

\section{Multicast}

Multicast communication is a mode of communication where one transmitter can be heard by many receivers. It is common in radio or satellite-based communication, but is even supported in digital communication systems such as local area networks (LANs). Though less common on wide area networks (WANs), recent multicast extensions to the Internet's protocol suite (IP) have spawned many research efforts to examine potential uses for wide-area multicast services. 


\subsection{Multicast and Broadcast on LANs and Multiprocessors}

Many LANs support some form of multicasting. The CSMA/CD, token-bus, token-ring, FDDI and ATM LANs, and even ground and satellite radio systems have multicast capabilities[1].

The IEEE 802.2 LAN addressing standards provide multicast protocol as well as broadcast protocol for CSMA/CD, token-bus and token-ring LANs[1]. On a CSMA/CD LAN (e.g., an Ethernet LAN), the sender of a multicast packet uses the group address for the destination address. This address is determined by the high-order bit of the destination address. If the high-order bit is a one, then the address is a multicast address. The rest of the packet is filled with the multicast address for the group. The broadcast address consists of all one bits. If the high-order bit is a zero, the address is unicast and only the machine with that address will accept the message.

Once the sender's packet is placed on the network, all hosts in the same LAN can see the packet. Each station will check the packet's address against their own and then against that of each multicast group to which it belongs. If there is a match, the packet is accepted by the receiver and processed similarly to that of a unicast message. The matching is usually done in hardware.

Multicast and broadcast are used in a variety of applications. Satyanarayanan, et al.[17] have developed a file system, Coda, for large-scale distributed computing systems using workstations. When measuring bytes transferred, they found that the use of multicast reduced the network load. Ahamad and Belkeir[13] present a multicast-based process for load balancing in an Ethernet-type LAN using the multicast capabilities of the local network. Satyanarayanan and Siegel[16] describe a parallel remote procedure call MultiRPC. MultiRPC does not use true multicast; software filtering of broadcast packets simulates multicast.

\subsection{Internet Protocol (IP) Multicast}

Numerous works contributed to the development of IP multicast and the Internet MBONE. ${ }^{1}$ In the $1984 \mathrm{RFC}^{2} 919$ [8], Mogul proposed broadcast protocols for besteffort IP datagram broadcasting. Internet gateways were used to protect against any unwanted broadcasts. His work includes information on how to broadcast IP datagrams on local networks that support broadcast, how to address broadcasts, and how gateways manage them.

In the 1985 RFC947[11], Lebowitz and Mankins presented a problem of multi-

\footnotetext{
${ }^{1} \mathrm{MBONE}$ is the Multicast backBONE of the Internet.

${ }^{2}$ Request for Comments
} 
network broadcasting and the motivation for solving the problem. They stated that a shortcoming of broadcasting was that broadcast packets are only received by hosts on the physical network on which the packet was broadcast. Their solution was to use a broadcast repeater transparently relaying broadcast packets from one LAN to another and to forward broadcast packets to hosts on networks which do not support broadcasting at the link-level. The forwarder and repeater were implemented separately. Lebowitz and Mankins stated that a large amount of overhead was involved.

Other works more recent and closely related to IP multicast are the 1988 RFC1075[6] Distance Vector Routing Protocol which provides a routing protocol for IP multicast that most MBONE routers use and the $1989 \mathrm{RFC1112[22]} \mathrm{Host}$ Extensions for IP Multicasting which is the recommended standard for IP multicasting in the Internet. RFC 1112 provides protocols for addressing, sending and receiving IP multicast packets. IP multicast provides unreliable (best-effort) delivery using UDP datagrams. IP multicast addressing is a key to multicasting in the Internet. IP multicast supports group communication in the Internet by using class D IP addresses. To travel within a LAN, the multicast address must be mapped to the local multicast address. Deering[22] details the procedure by which an IP host group address is mapped to LAN multicast addresses in order to send IP multicast datagrams into the LAN. In order to map an IP group address to an Ethernet multicast address, the low-order 23-bits of the IP address are placed into the low-order 23 bits of the Ethernet multicast address. Multicast routers control the forwarding of IP multicast datagrams between any two subnets supporting IP multicast.

Many IP multicast routing algorithms exist [24]. Currently DVMRP [6] is used to route multicast datagrams through the MBONE. IP multicast routers (mrouters) are necessary for routing packets through non-IP multicast routers; this is termed tunneling. Unlike unicast datagrams which could pass through any router in its path, an IP multicast packet must be encapsulated using the address of another mrouting host as the destination. The IP multicast packet can only travel from one subnet to another subnet if both subnets contain a working mrouter where both are directly connected or are connected via the MBONE. Being directly connected means that the packet is forwarded from the mrouter of one subnet to the mrouter of another subnet. Being connected through the MBONE means that there are one or more mrouters through which the packet passes other than the mrouters in the sender's and the receiver's subnet. All IP multicast routes are built manually.

IP multicasting often involves audio and video data which is bandwidth in- 
tense. The MBONE tools offer a non-traditional interactive teleconferencing media such as Network Video ( $n v / v i c$ ), Visual Audio Tool ( vat) and a shared drawing whiteboard $(w b) . N v$, vat, and $s d$ use unreliable IP multicast; $w b$ provides its own reliable IP multicast protocol. As the number of IP multicast-capable machines grows, the need for applications which take advantage of IP multicast will also grow, and this can be a problem. According to Jacobson[5], "The MBONE has a total of $500 \mathrm{~kb} / \mathrm{s}$ of bandwidth that has to be shared between 1200 networks and 10,000 hosts. To put that in perspective, $500 \mathrm{~kb} / \mathrm{s}$ is a total of 6 vat conversations or $4 n v$ video sessions."

\subsection{Reliable Multicast}

Deering[22] states, "A multicast datagram is delivered to all members of its destination host group with the same best-efforts reliability as regular unicast IP datagrams." In building fault-tolerant software such as PVM, best-effort reliability will not suffice, especially if one multicasts beyond the subnet where, the frequency of packet loss increases. In this section, literature about reliable multicast is reviewed.

Pingali, et al.[23] compare three reliable multicast protocols. The first protocol is a non-optimized positive acknowledgment-based protocol. The second protocol is a non-optimized negative acknowledgment-based protocol which uses unicast NAKs. The third protocol is a NAK-style protocol which Ramakrishnan and Jain developed for LANs and which Jacobson developed for WANs. This third protocol uses a multicast (or broadcast) NAK to reduce the amount of reverse-traffic.

In the ACK algorithm, the sender initiates the retransmission by timing-out while waiting for acknowledgments. As the number of receivers increases, the number of ACKs increases. Not only does this cause contention in the network, but the sender must take time to process each ACK. This protocol was found to have a lower throughput than the two negative acknowledgment protocols. However, it is much less complicated to implement.

In the NAK versions, the receiver initiates error control. As mentioned, the NAK protocols differ in the manner in which a NAK is returned. In the nonoptimal unicast NAK protocol when a host receiving multicast packets detects an error (e.g., a lost packet), a NAK is immediately sent to the transmitting host for which the error was detected. Using a NAK decreases the reverse traffic since a NAK is only sent when an error is detected.

In the multicast NAK protocol, a random time is set between discovering an error and sending a NAK. Before sending a NAK, the receiver checks whether or 
not the NAK has been multicast by another receiver. If so, it sets a timer and waits for the retransmission, else it sends the NAK. The NAK is sent via multicast to all members of the group. This decreases the number of NAKs being sent by receivers from as many as $N$ down to at most one per multicast. According to Pingali et al., Jacobson has tried using the multicast NAK-style algorithm in a shared whiteboard. Of these three protocols, the multicast NAK protocol was found to have the highest throughput;

Hall [9] summarizes a number of applications that include multicast services, including VMTP [4], XTP [26], ISIS [12], MTP [20], MTP2 [2], RAMP [18], and Ameoba [14]. Of particular relevance, Huang, et al.[3] developed a reliable multicast transport service for an Asynchronous Transfer Mode (ATM) network. This service sits atop the unreliable ATM Adaption Layer 5 (AAL5) and uses the vendor supported Application Programming Interface (API)'s socket-like services, not IP. ATM relies on fast packet switching for speed. ATM multicasting is based on parallel transfers of $N$ copies of the packet to be multicast. Since this method is somewhat unreliable, Chang, et al.[21] investigate reliable messagepassing protocols; PVM was chosen for their tests. An ATM LAN with ATM switches connecting workstations was used in their tests. One performance test involved a normal implementation of PVM over an Ethernet network using BSD sockets. A second test involved implementing PVM in an ATM network using BSD sockets. The third and fourth tests involved implementing PVM in an ATM network using different versions of the Fore Systems' ATM API library (FSAA) with the necessary changes to PVM. FSAA which provides the socketlike interface for ATM messages is a lower-layer protocol than BSD sockets. FSAA is a connection-oriented service in which the connection must be made before data is exchanged.

Although the multicast process was altered for the tests, PVM's acknowledgment protocol remained the same. In phase I of each test, a task ID list is sent to each remote $p v m d$. The list contains the IDs for all tasks on the same host as the remote $p v m d$ which are to receive the multicast packet; this is normal for PVM. In phase II, first normal PVM procedures were used to multicast the data; $N$ serial sends were sent to $N$ remote pvmds. Then PVM was re-implemented such that $N$ simultaneous sends were made to $N$ remote pvmds. In the serial version of phase II, all ATM/PVM tests had a throughput of about $7 \mathrm{Mbps}$; the Ethernet/PVM test had a throughput of about $4 \mathrm{Mbps}$. In the parallel versions of phase II, the ATM/FSAA/PVM tests provided the highest throughput of about $27 \mathrm{Mbps}$, which is about half the maximum throughput of the ATM/FSAA system alone. The ATM/BSD/PVM test had a throughput of approximately $20 \mathrm{Mbps}$. 
The Ethernet/PVM test had a throughput of about $8 \mathrm{Mbps}$.

\section{Reliable IP multicast in PVM.}

The need to solve scientific problems quickly has led to the development of parallel computing[10]. Many parallel computers use multiple processors connected by very short communication links all within one frame. Parallel computers allow parts of a problem (tasks) that can be executed independently of each other to be distributed over many processors. Message-passing routines are used for communication among the processors. In general, parallel computers are expensive. Due to the demand for a less expensive parallel computer and the development of efficient, inexpensive, high speed computers (e.g., workstations) and networks, distributed operating systems were developed to run on LANs. Within a distributed operating system, hosts use message-passing routines to communicate. A group of computers connected by a distributed operating system work together to form a multiprocessor. In addition to workstations, a distributed operating system may be used to connect mainframes and/or multiprocessors.

The Parallel Virtual Machine (PVM) is a message-passing system which works like a distributed operating system, but usually it runs on top of the existing operating systems (e.g. Unix)[19]. PVM does not provide a file system or memory manager. Since PVM joins physically separate and architecturally different machines over a LAN or a WAN, it is called a virtual machine. The hosts in the PVM do not have to be in the same LAN, but there does have to be some type of internetwork between them in order to pass messages.

The PVM library (libpvm) allows user applications (tasks) written in $\mathrm{C}$ or FORTRAN to communicate with the local PVM daemon ( $p v m d$ ) or remote $p v m d s$ and remote tasks easily. Most communication between tasks is relayed via pvmdto-pvmd communication. The libpvm provides an interface between the task and the pvmd. In addition to routing and controlling messages, the pvmd provides authentication, process control and fault detection[19]. The first pvmd that is started manually by the user is the master. The master can add, delete, or reconfigure slaves; other than these jobs, the slaves function like the master.

PVM version 3.2.6 provides a multicasting routine, pvm_mcast(). This routine forwards the receivers' task identifiers, TIDs, to the local pvmd and then receives back the group identification, GID. The function then forwards the multicast message with the GID to the local pvmd. This pvmd first sends the TIDs and the GID to the appropriate pvmd, and then sends the message via unicast messages to those pvmds (see section 4.2.2 for further details). Also, PVM provides a special 
library, libgpvm, for group communication. This allows the user to identify groups by a number from 0 to $(p-1)$ where $p$ is the number of tasks. To send to a group, the user calls pvm_bcast() using the group identification, GID. The pvm_bcast() relays the message to the local pvmd. This pvmd in turn processes the incoming request in the same manner it would a pvm_mcast() request.

Several mechanisms for delivering multi-daemon-destination messages have been considered by the PVM development group. Broadcast has been examined as perhaps a more efficient method in sending a multi-destination PVM message than a one-to-all unicast method. The results of the broadcast study were very good especially for larger message and receiver set sizes [25]. However, with a broadcast, all machines on the LAN, even those not using PVM, receive the packet. Broadcast is also limited to hosts on a LAN and does not work over a WAN. Some PVM version 2's use a spanning-tree algorithm much like that used by the hypercube in order to perform multicasting. However, later versions (3.0-3.3) returned to the one-to-all fanout approach to perform more robustly. IP multicast permits a single message to reach a subset of hosts on both a LAN or a WAN. If the host is not a member of the multicast group, it ignores the message. So, IP multicast should be a suitable delivery mechanism for PVM.

IP multicast was implemented reliably in PVM and was compared to the unicast-based function pvm_mcast() which is currently used for multicasting in PVM. The objective was to understand the issues involved in using reliable mul-

ticast protocol in a real application and to measure and analyze any performance improvements in reliable IP multicast.

\subsection{Multicast in PVM}

\subsubsection{PVM message-passing}

At the application level, sending a PVM version 3.2.6 message consists of three steps. The task must first initialize a send buffer by a call to pvm_initsend() or pvm_mkbuf(). Then, the task must pack the message into the buffer using one or more PVM packing routines (i.e., pvm_pk*()). Finally, the buffered message is sent to another task by either calling the pvm_send() routine for unicasting or the pvm_mcast() routine for multicasting.

Receiving a PVM message consists of at least two steps. Optionally, one may use pvm_bufinfo() to receive information about a particular message. To actually receive the message, either pvm_recv() or pvm_nrecv() (a non-blocking receive) can be called. Then, the received message must be unpacked by using pvm_upk*().

Both the pvm_send() and pvm_mcast() routines communicate with remote $p v m d s$ and tasks by first sending the message to the local pvmd via Unix domain 
datagram socket (see Figure 3.1). The message is then sent to the appropriate receiving $p v m d(\mathrm{~s})$ via a UDP socket. The message is then sent to the remote pvmd and finally to the appropriate task(s) on the same host. The sending $p v m d$ retransmits the message until it receives an ACK or until the number of retries reaches the maximum. Upon receipt of the ACK the message is removed from the output queue. If the message is not ACKed by the last re-try, the message is dequeued, and the remote pvmd is assumed to be dead. The sending pvmd informs all other pvmds of the problem, and the downed pvmd is deleted from the PVM host tables.

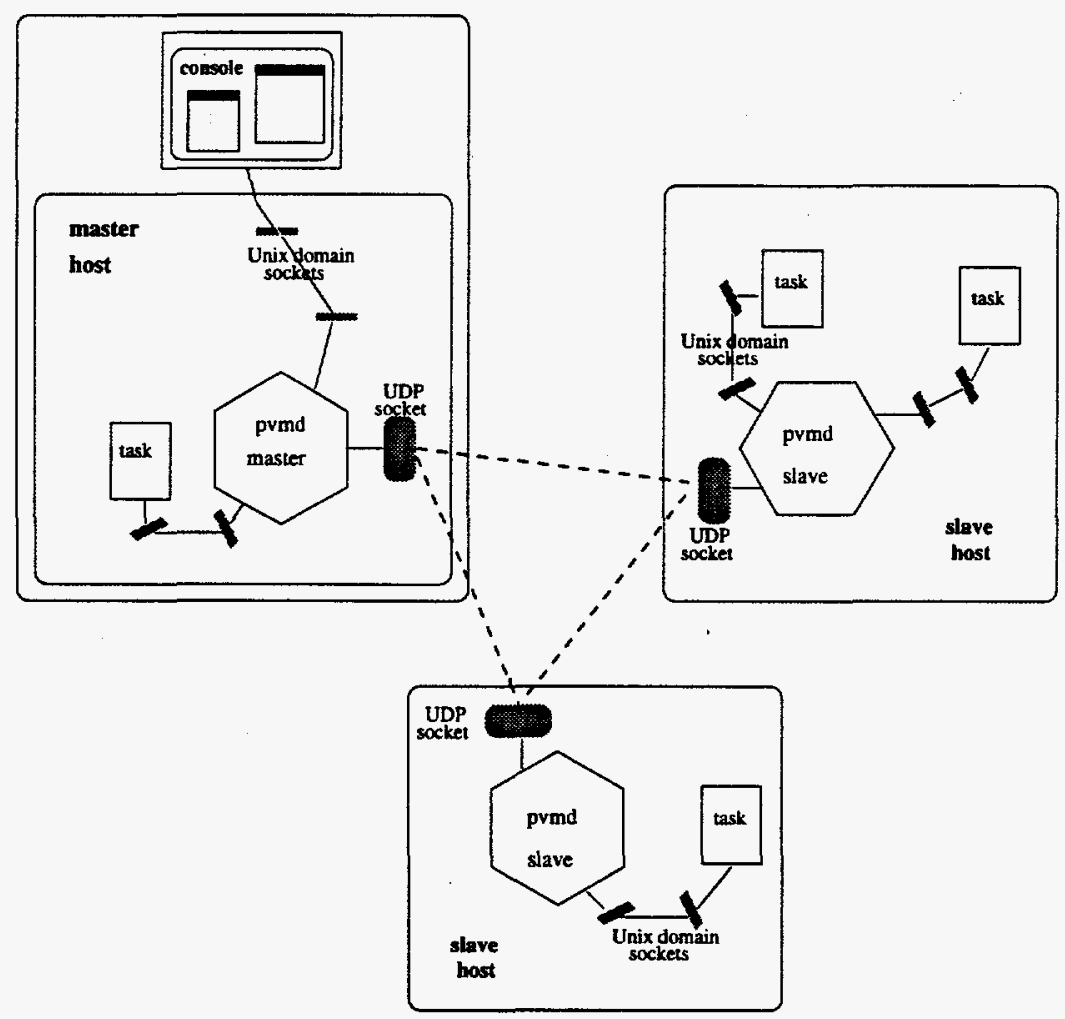

Figure 3.1: A Partial Anatomy of PVM.

The receiving $p v m d$ replaces the ACK number with the sequence number and returns an ACK for the packet to the sending pvmd. The receiving pvmd will then forward any messages destined for tasks on its host. A task may receive a message by calling a receive routine and then unpacking each of the packed items. The message may be received from a specific source with a specific message tag, or from any source with a specific message tag, or from a specific source with any message tag. 


\subsubsection{PVM's pum_mcast() protocol}

The function pvm_mcast(), a part of the libpvm, is used by a task for multicasting a message. PVM version 3's daemon uses multi-unicasts (a one-to- $n$ fanout multicasting algorithm) to reliably send a task-initiated message to multiple destinations. Some earlier versions use a spanning-tree algorithm. In this section, the following are discussed: forming a multicast address, initiating a multicast message, and sending a multicast message in PVM. The decision for choosing the one-to- $n$ fanout algorithm is also discussed.

\subsubsection{PVM multicast address}

Within the PVM system, a task identifier (TID) is used to address tasks, groups of tasks, and pvmds. With its $G$ bit set to one, the TID represents a group address or GID. The GID is formed by the pvmd. A new GID is formed for each multicast message; any discarded GIDs may eventually be reused. The multicast descriptor, struct mca, stores information about all active multicasts. As with all TIDs, the $H$ field is set to the value of the host index for the local host. The $L$ field is set to the value of a counter which is incremented for each multicast. The main difference between a non-multicast task identifier and a GID is the setting of the $\mathrm{G}$ bit.

\subsubsection{Initiating multicast}

The multicast message is initiated by a request with a code of TM_MCA sent from the task via libpvm's pvm_mcast() to the local pvmd. The request contains a list of task identifiers, the multicast group, for all tasks (recipient-tasks) which are to receive the multicast message. Thus, PVM uses a closed group scheme. This list can change each time a new multicast message is sent making the groups dynamic. However, once the TM_MCA message is formed, no other task may join the group. The message is passed to the pvmd's tm_mca() function which forms the GID and a new mca. Also, the list is sorted according to the host index and checked for duplicate or erroneous TIDs. The information is stored in the mca. The pvmd replies with the GID to the requesting task, and then sends a message with a code of DM_MCA to remote pvmds (recipient-pvmds) which have tasks in the group. The DM.MCA message contains the GID and the TIDs of tasks in the multicast group which are on the same host as the recipient-pvmd. The receiving $p v m d$ 's dm_mca() function processes the information and each forms a local mca in order to know which tasks are to receive the multicast message (see Step I of Figure 3.2). 
Step I:

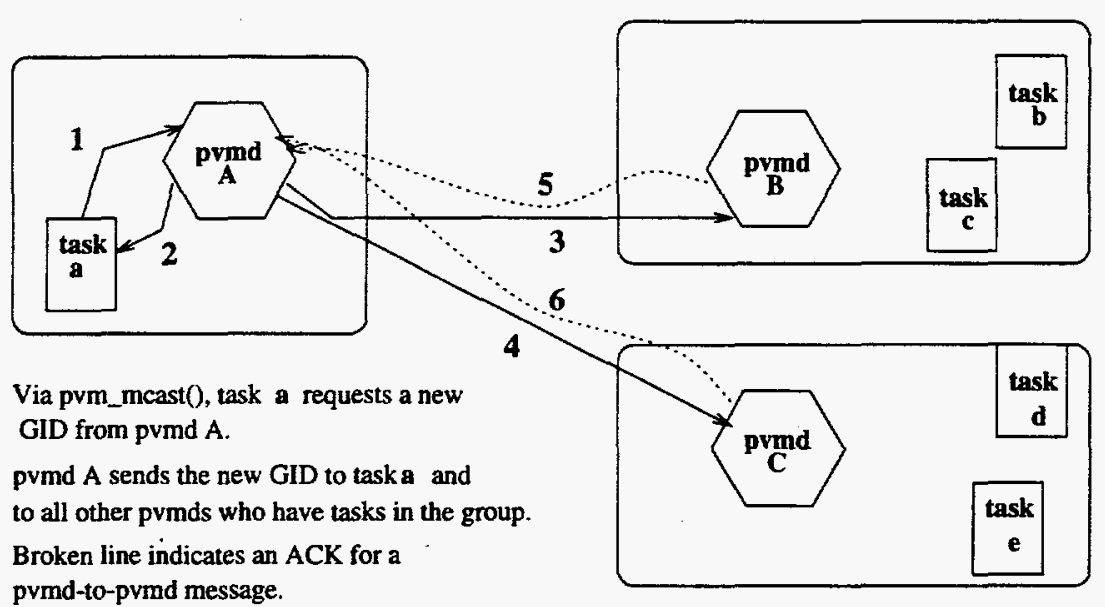
pvmd-to-pvmd message.

\section{Step II:}

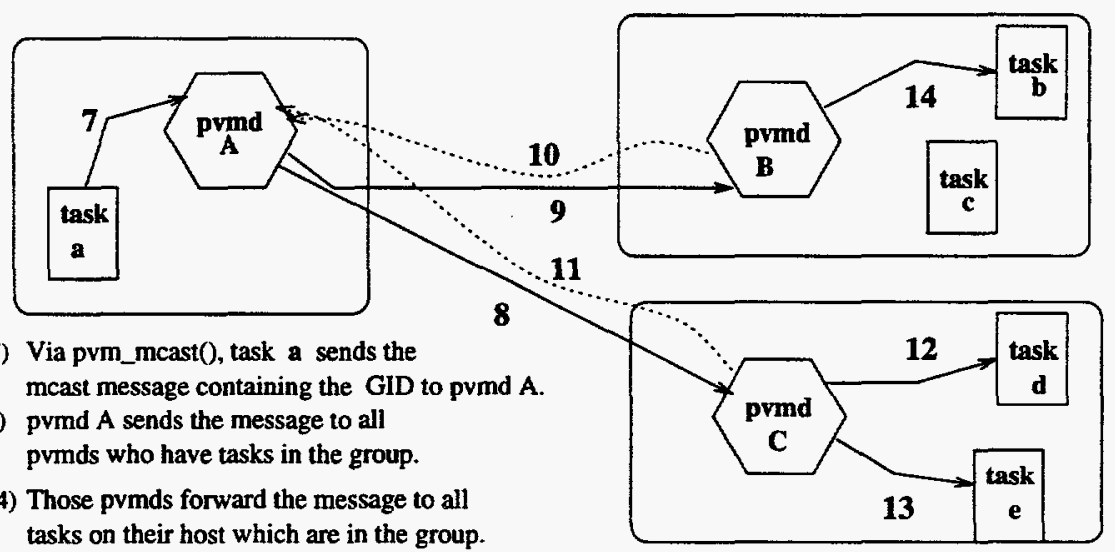

$(12,13,14)$ Those pvmds forward the message to all tasks on their host which are in the group.

(10,11) Broken line indicates an ACK for a pvmd-to-pvmd message.

Figure 3.2: Diagram of PVM's pvm_mcast() function. 


\subsubsection{Sending multicast}

Upon receipt of the GID, pvm_mcast() then sends the multicast message to the local pvmd, using the GID (see Step II of Figure 3.2). When the local pvmd receives the message from the sending task, the routing layer of the $p v m d$ prepares a packet descriptor, struct pkt, one for each local recipient-task and one for each remote recipient-pvmd. The pkt stores information, for use in timing out, sequencing, and acknowledging the message as well as a copy of the message. Each message is placed on a queue. PVM then unicasts each message to the proper recipient. When a multicast packet arrives at a recipient-pvmd, it is ACKed by the pvmd, and it is then duplicated and sent to each recipient-task on the same host (see Step II of Figure 3.2). When these pvmds detect the packet with the end-of-message (EOM) bit set, they remove the mca. Because of PVM's reliability measures, the multicast address and data packets will arrive in proper order at each destination.

\subsubsection{PVM Multicast routing}

A spanning-tree algorithm was used in some Version 2 packages to deliver multicast messages because it decreases the contention on the Ethernet for a particular host. The spanning-tree divides the sends among the hosts in the PVM. Therefore, while one host may be waiting to send, another host can send. To implement the spanning-tree algorithm, each pvmd is identified by a node number from 0 to $(n-1)$, where $n$ represents the number of nodes. A bit-wise operation on the node number determines the children of each node as is done in the Hypercube. The ACKs were sent directly to the root of the tree.

There were several drawbacks to using a spanning-tree in PVM. For instance, extra logic is required to handle the case when a machine on the interior of the tree goes down. Extra work is involved in determining who did not receive the multicast message. One must determine whether the node failed before or after a multicast was sent. One must determine whether all, none, or part of the children received the message. Also, a recovery process involving eliminating the failed machine and eventually re-sending the message to those who lost it must be completed. Due to its robustness, the one-to- $n$ fanout was re-implemented in PVM version 3. This ensures that the failure of one host will not cause the loss of messages except for the ones to that host. Also, fault-tolerance is simplified. 


\subsection{Adding IP multicast to PVM}

As noted in section 2, efficiently implementing reliable multicast is a matter of open research. There are advantages and disadvantages to both NAK-based and ACK-based reliablity models. Since a positive acknowledgement system already exists in PVM's pvmd-to-pvmd communications, it was decided to use that system to implement reliable IP multicast in PVM.

\subsubsection{The pvm.jpmcast() protocol}

Reliable IP multicast was added to PVM with pvm_ipmcast(). It was hoped that using IP multicast would decrease out-going traffic and contention, and improve PVM's performance for multicasting. Just like pvm_mcast(), pvm_ipmcast() is part of libpvm, and works with the pvmd to send a single message to multiple destinations. Only a slight difference between the two functions exists in the libpvm. Most of the changes were made to the pvmd. In the following section, implementation issues are discussed, such as making the IP multicast socket, initiating an IP multicast message, sending an IP multicast message, and acknowledging the message.

\subsubsection{IP multicast socket}

In PVM's mksocs() routine in startup.c, several sockets are made. The netsock socket is used for pvmd-to-pvmd communication. The ppnetsock socket is used for $p v m d$-to- $p v m d^{\prime \prime}$ communication where $p v m d^{\prime \prime}$ is a daemon process spawned by the master pvmd in order to start slave pvmds. The loclsock socket is used for task-to-pvmd communication. The mnetsock socket was added for pvmd-to-pvmd IP multicast communication. Now, the pvmds not only have to listen to the usual sockets, but also must listen to the IP multicast socket.

The IP multicast loop-back option was set to zero, which means the sender will not receive its own multicast messages. The sender must know what address and port number on which the receiver will be listening. In this study, the IP multicast address and the port numbers were static. For testing purposes, this was helpful, since one would always know what address and port number to watch while using network debugging tools. However, for general use, this will cause problems, especially if two users on the same network choose the same values.

\subsubsection{Initiating IP multicast}

Pvm_mcast() was not removed from the libpvm. Most of the changes were made to 
the pvmd. The pvmd can distinguish between those packets to be sent via one-to$n$ fanout from those to be sent via IP multicast. As for the libpvm, pvm_ipmcast() is virtually the same as pvm_mcast(). However, instead of sending the TM_MCA code to the local pvmd to request a GID, pvm.ipmcast() sends the TM_IP_MCA code. This allows the pvmd to differentiate between the two types of multicast messages. The task identifiers contained in the request are sorted and checked as before. Also, the GID is returned to the initiating task and the DM_MCA message is sent to recipient-pvmds as before.

\subsubsection{Sending IP multicast}

In order to satisfy PVM's design criteria, IP multicast was implemented reliably. In this implementation, IP multicast was inserted in such a way that few changes were made to PVM's logic. Most of the changes were made to the PVM routines loclinpkt(), netoutput() and netinput() found in pvmd.c. Instead of duplicating the ACK process, the ACK protocol of PVM was changed slightly to be used for the IP multicast acknowledgments needed by a reliable multicast protocol. This kept the changes to PVM modest, which was preferable.

In the loclinpkt() function, in addition to the normal pkt descriptors for unicast copies (subsequently denoted by pkt $_{u}$ ), a pkt (subsequently denoted by pkt $\mathrm{m}_{m}$ ) was formed whose packet GID $H$ field was set to the local host index. This pkt t $_{m}$ was used as the IP multicast packet descriptor. The local pvmd controlled the removal of the IP multicast packet from the queue by setting the appropriate $A C K$ information for this pkt $_{m}$ when necessary.

While the other pkt $_{u} \mathrm{~s}$ were being formed, a descriptor (struct ip_info) stored a pointer to the sequence number for each remote pvmd. As well, when the IP multicast pkt $_{m}$ was formed, information (e.g., the GID, an IP multicast counter, and an ACK counter) was recorded in the ip_info. The pkt ${ }_{u}$ s containing unicast copies were formed as they would be in pvm_mcast() with a couple of changes. In case more than one task was multicasting from a particular host, each of these $\mathrm{pkt}_{u}$ s contained an identifying number, $p k_{-} m c a_{-} n u m$. This allowed the ip_info to be updated correctly when a multicast packet was ACKed. As well, an IP multicast marker, $p k_{-} i p m c a$, was set to one so that the netinput() function could determine if the ACK was for an IP multicast packet.

These $\mathrm{pkt}_{u} \mathrm{~s}$ were used not only to store the ACK information, but were also used as back-up $\mathrm{pkt}_{u}$ s to the IP multicast $\mathrm{pkt}_{m}$. If the $\mathrm{pkt}_{m}$ packet was removed from the queue before a back-up $\mathrm{pkt}_{u}$ was ACKed, this back-up $\mathrm{pkt}_{u}$ packet was sent as it would be in pvm_mcast() via unicast to the intended receiver.

Presently, due to the way in which the unicast back-up $\mathrm{pkt}_{u} \mathrm{~s}$ were allowed to 
collect the ACK data, the appropriate sequence number had to be sent to the recipient-pvmd in order for the ACK to be formed properly. This information was sent in the multicast packet. In the netoutput() function of the $p v m d$, when the IP multicast packet was encountered, the sequence numbers for the unicast packets were copied from ip_info to the tail of the packet header. The header size had to be increased for this, and this limited the number of receivers which could be used. Then the packet was sent using the IP multicast address and port numbers on the mnetsock socket (see Figure 3.3). If an ACK was received for a multicast packet, then a zero was placed in the ip_info's sequence number slot for the ACK's sequence number. The unicast copies held in the $\mathrm{pkt}_{u} \mathrm{~s}$ were only sent if the IP multicast packet timed out.

The recipient-pvmds listening for incoming packets would receive the IP multicast packet on their mnetsock. This was how the recipient was able to tell that the incoming packet was an IP multicast packet. When the netinput() function was called, the sequence numbers were first removed from the tail of the header of the packet. The number belonging to the particular pvmd was stored as the sequence number for the incoming packet. If the new sequence number was zero, the function returned to its calling function decreasing the amount of reverse traffic, else the packet was processed as it would be in the non-IP multicast routine.

If the IP multicast message was still in the queue when netoutput() was called again, the function first checked the ip_info to determine whether or not the multicast packet had been successful; then it checked to see if the number of multicast retries $(M)$ was greater than the maximum number of retries $(R)$. If both were false, the IP multicast packet was re-sent. However, if either was true, the descriptor's information for that packet was flushed, and the packet was $A C K e d$ by the local pvmd. Any remaining unACKed back-up packets were sent via unicast at this point.

\subsubsection{Acknowledging IP multicast}

Upon receipt of an ACK, the pvmd checked to see if the ACK was for an un$A C K e d$ packet in the queue. If so, then the $\mathrm{pkt}_{u}$ that was to receive that $\mathrm{ACKs}$ information was checked to determine whether or not it was an IP multicast packet. If it was, then the ip_info ACK information for this particular IP multicast packet was incremented in order to keep track of the ACKs. This was how the IP multicast packet could detect how many ACKs had been received. 
Step I:

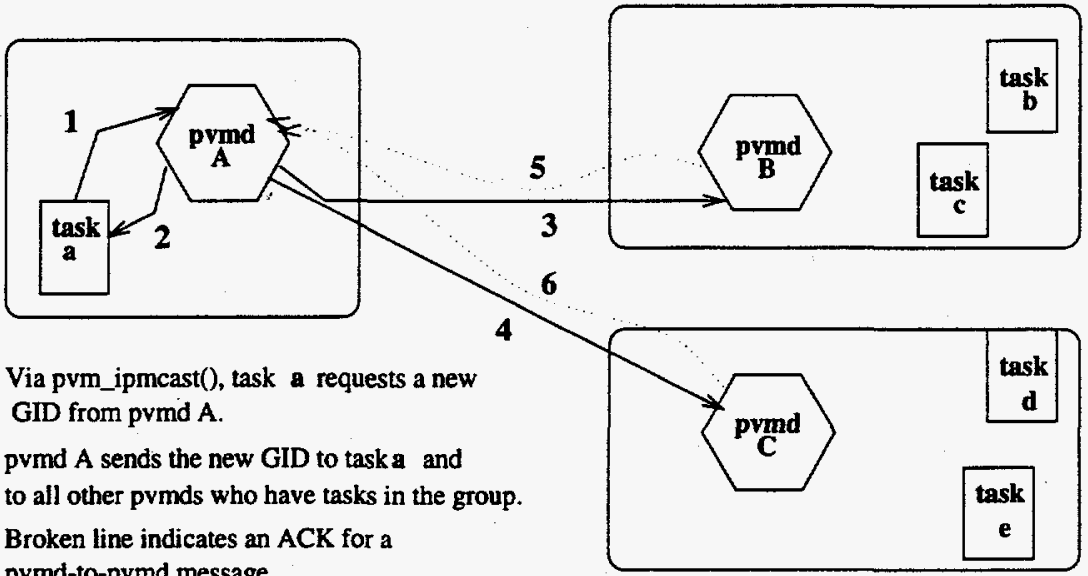
pvmd-to-pvmd message.

Step II:

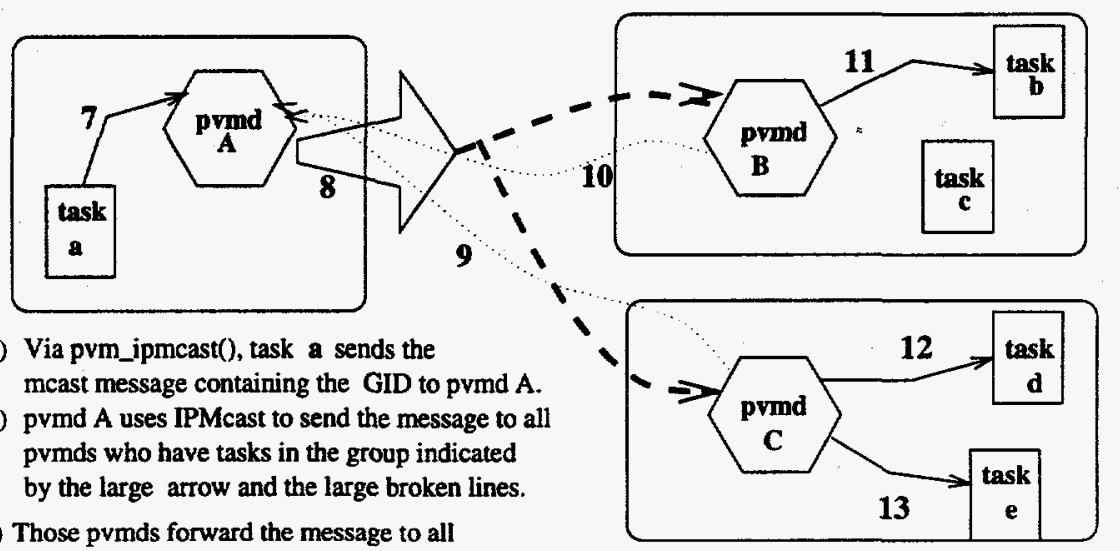

$(11,12,13)$ Those pvmds forward the message to all tasks on their host which are in the group.

$(9,10)$ Small broken line indicates an ACK for the multicast message.

Figure 3.3: Diagram of the pvmipmcast() function. 


\subsection{PVM IP multicast Performance}

The goal for implementing reliable IP multicast in PVM was to determine whether it would improve performance, particularly for larger message sizes and a larger number of receivers. The ACK-based protocol was tested without PVM on both local and wide-area networks [9], and a performance model was developed to predict speedup of multicast communication over unicast. The testing illustrated that performance was sensitive to packet size, network load, and multicastrouting hops $(t t l)$ [9]. The tests of the reliable multicast protocol and the model suggested that reliable IP multicast should provide better communication performance than a unicast-based multicast. However, the performance of the IP multicast, pvm.jpmcast(), function that was developed as part of this study was slightly worse on average for the LAN when compared to PVM's multiple unicast version, pvm_mcast(), and the IP multicast version for the WAN was much worse. As the number of hops through the Internet increased, the performance of the reliable IP multicast in PVM deteriorated greatly.

The performance problems mentioned above and several factors mentioned below may have led to decreased performance of the reliable multicast protocol in PVM. For instance, the DM_MCA message must be accepted by a receiver before the IP multicast packet can be accepted. Therefore, the IP multicast packets are hampered by these initial unicast control packets. Also, the IP multicast process incurs overhead in addition to the normal PVM packet processing. For instance, the ip_info IP multicast descriptor must be properly filled for each multicast message, and the ACKs for multicast packets must be counted so that necessary retransmissions occur properly. In addition, the process that was used for determining a retransmission method may have caused unnecessary waiting. As well, the receivers of a multicast packet must check the packet header for the appropriate sequence number to determine whether to continue processing the packet. Due to this, IP multicast in PVM does not conform to our model except that as the number of receivers increases time increases at a slower rate for IP multicast than for multiple unicasts.

\subsubsection{LAN Performance of pvm_ipmcast()}

On the LAN using 2, 4,6 or 8 receivers each at UTK/CS and at ORNL and a 1024-byte message, the pvm_mcast() messages are faster than the pvm_ipmcast() messages on average by 2 to $20 \mathrm{~ms}$ (see Figure 3.4). Also, comparing these results to the standalone reliable multicast protocol test [9], both IP multicast and multiple unicast methods incur overhead due to PVM. The additional IP multicast overhead and restrictions appear to decrease the IP multicast performance 
further.

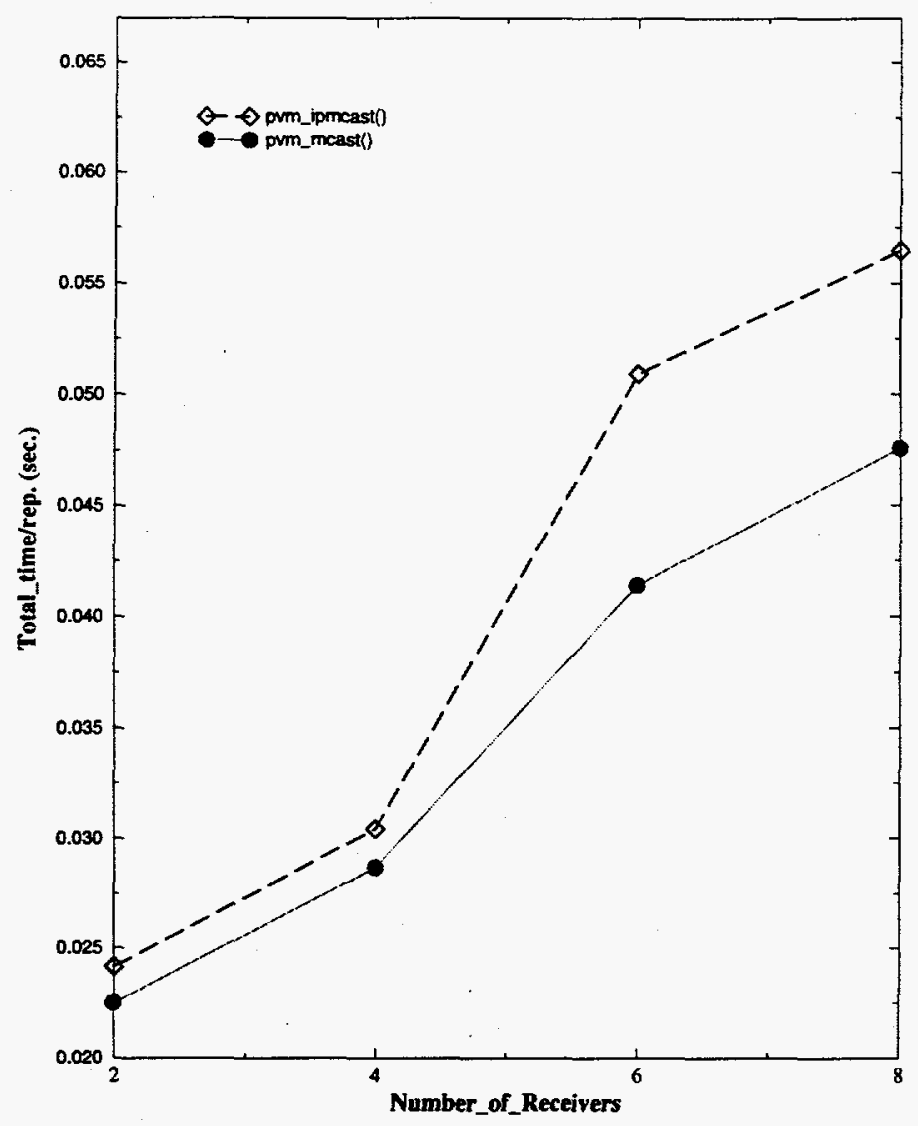

Figure 3.4: pvm_ipmcast() vs pvm_mcast() LAN Performance Total Time/rep (sec.) vs Number of Receivers for 1024-byte packets.

\subsubsection{WAN Performance of pvm_ipmcast()}

On the directly-connected WAN using $1,2,3$ or 4 receivers each at UTK/CS and at ORNL and a 1024-byte message, PVM's pvm_mcast() outperformed the pvm.jpmcast() by 5 to $10 \mathrm{~ms}$ for a $t t l$ of 18 (see Figure 3.5) and by about 400 $\mathrm{ms}$ for a $t t l$ of 76 (see Figure 3.6), except in the case of eight receivers where they perform about the same. For the IP multicast version the increase in time as the number of receivers increases is a slower rate than that of the non-IP multicast version. In these PVM WAN studies, IP multicast began much worse than multiple unicast, but as the number of clients increased, the difference in their performance decreased. For the WAN, not only do the problems mentioned in the subsection above exist, but also the numerous hops through the MBONE 
and it's mrouters add to the latency for large ttls. Due to this, comparisons between IP multicast via the MBONE and multiple unicast in a WAN may not always be fair at the present time.

Since PVM is to be used as a parallel machine, speed is a major factor. The implementation of the reliable IP multicast protocol in PVM does not provide the speed-up which was desired because only the multiple unicasts were replaced with IP multicast and not much else was altered. Some of the reasons for limited IP multicast performance are examined in the next section.

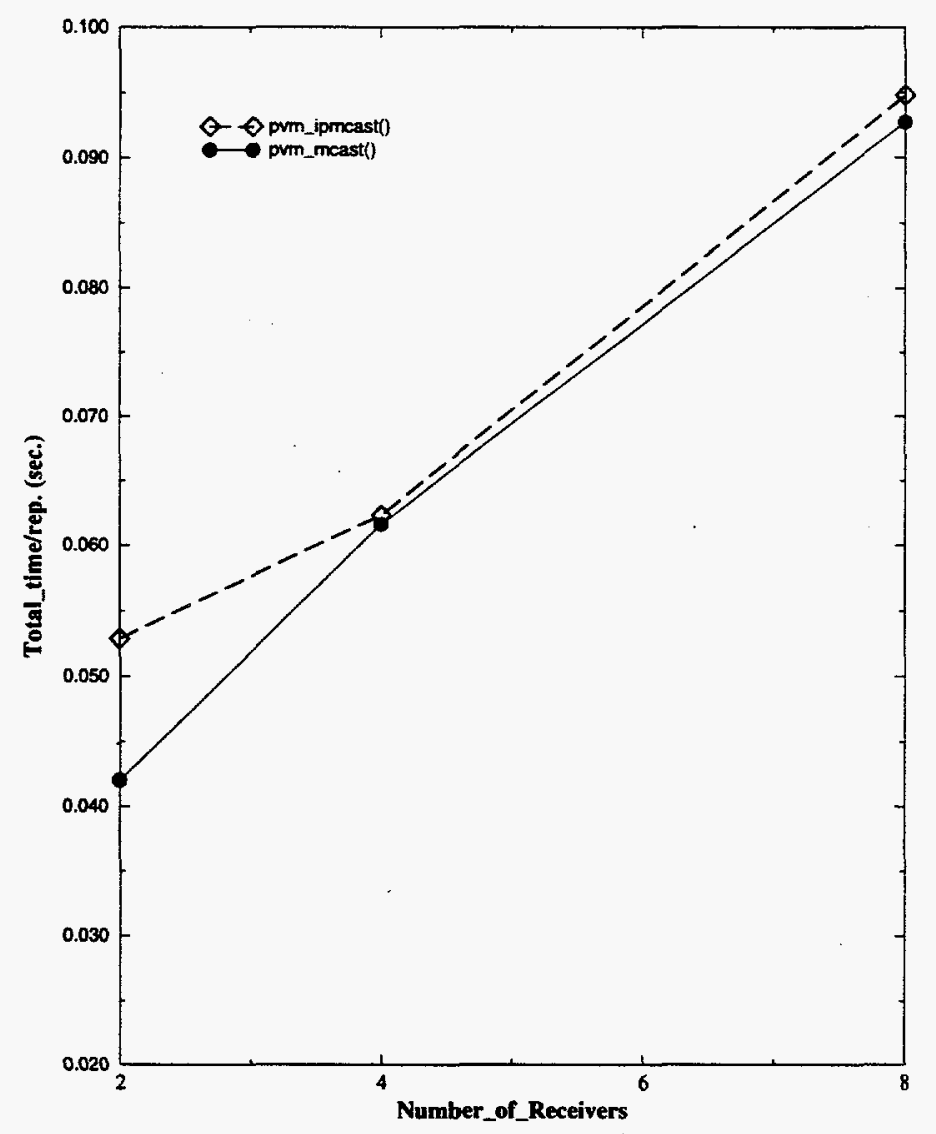

Figure 3.5: pvm_ipmcast() vs pvm_mcast() WAN Performance (ttl $=$ 18)

Total Time/rep (sec.) vs Number of Receivers for 1024-byte packets. 


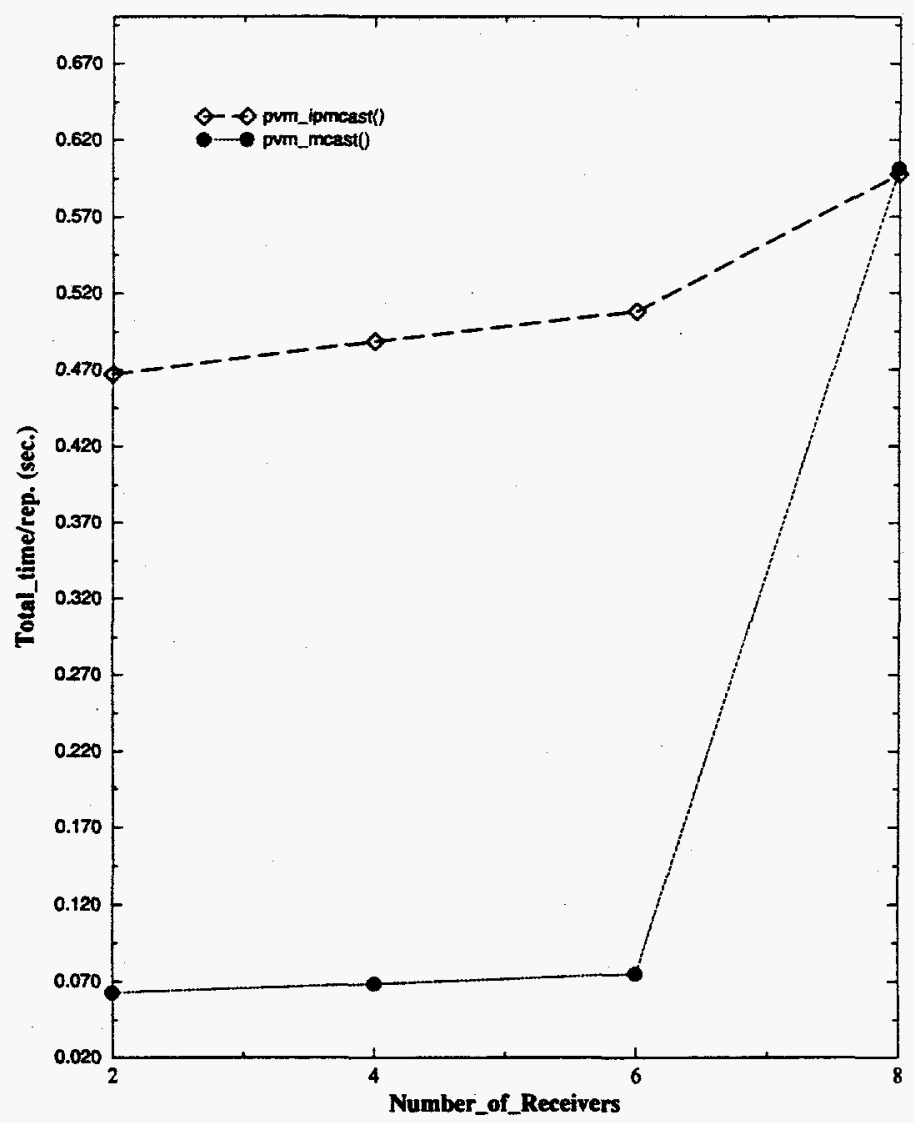

Figure 3.6: pvm_ipmcast() vs pvm_mcast() WAN Performance $(t t l=$ 76)

Total Time/rep (sec.) vs Number of Receivers for 1024-byte packets. 


\section{Summary}

Although many view the MBONE as a production model, there are still many problems to be solved and new uses to be found. In this research, several questions about reliable IP multicast were addressed. Those findings are reviewed below, as well as limitations and future work in IP multicast.

The machines used in the test environment had to be IP multicast-capable. Since none of the machines in the test configuration came with kernel support in place, each machine had to have its kernel rebuilt using the IP multicast software. The use of the IP multicast socket options were described for setting the maximum hop count $t t l$ and for joining the multicast group. In addition, each subnet had to have an mrouter for forwarding packets. Multiple mrouters on the same LAN caused long periods of packet loss. Currently, the routes are implemented manually via the /etc/mrouted.conf file.

Problems relating to the WAN used in the performance tests were discovered during these experiments. Part of the problem is believed to be caused by DVMRP multicast routers located on hosts at UTK/UTCC and UTK/CS that are connected by a bridge. Also, since IP multicast is fairly new, bugs still exist in some of the software. Another problem had to do with the number of hops through the MBONE, which decreased performance. Currently, the IP multicast performance over a WAN is limited by the number of hops. For each hop, there is an increased probability that a packet may be lost. An IP multicast packet traveling from one subnet to another may have to travel many times further than a unicast packet would between the same subnets. As the number of mrouters increases and the IP multicast path is shortened, this should not be as much of a problem.

Many reliable multicast protocols exist. However, most protocols have been developed for LANs where the kernels of the machines were altered or for specific multicast programs, such as Jacobson's whiteboard program. Therefore, the reliable multicast protocol in this study was written keeping in mind that it would be implemented in PVM. Several items that exist in PVM were not duplicated during the implementation of the reliable multicast protocol, such as special addressing for multicast packets, ordering of packets, and task protocol. Also, PVM's positive acknowledgment protocol was used as the basis of the ACKs for the reliable multicast protocol.

Finally, the performance of reliable IP multicast was examined. Reliable IP multicast worked well in the LAN used in this study. For larger packet and receiver set sizes, IP multicast was much better than multiple unicasts. However, as the number of multicast-router hops increased on the WAN, IP multicast 
performance deteriorated. The performance of IP multicast in PVM was also disappointing. In order to implement the reliable multicast protocol in PVM and to obtain an improvement over what already exists, major changes to PVM's logic would need to be made, such as providing an IP multicast sequence number that would allow only the IP multicast packet's pkt to receive all the multicast ACK information. When the IP multicast pkt is dequeued, only the unicast back-up pkts that are needed for unicast retransmissions would need to be formed. In PVM, there also needs to be a process for discovering mrouter problems. In the case where an mrouter is down, all pvm jpmcast() requests could automatically be processed as pvm_mcast() requests. In addition, when the number of IP multicasts to a virtually static group is large, providing a less dynamic multicast group would decrease the number of DM_MCA messages and could decrease any delays due to a receiver discarding an IP multicast packet which could arrive before the DM_MCA message. As the single echo tests showed [9], IP multicast improves over multiple unicasts as the number of receivers increase. It is possible PVM pvm_ipmcast() performance would have surpassed pvm_mcast() with more than eight receivers, but the resources to conduct such tests were not available.

\subsection{Limitations}

There are several drawbacks to using IP multicast. Many of the problems are related to management, privacy, and reliability. Another issue is that IP multicast is not widely available. Also, no easy manner exists in which IP multicast addresses and port numbers can be generated through the application. In addition, there are major steps yet to be made in the routing of IP multicast and in increasing its bandwidth. These issues are discussed further in the following sections.

\subsubsection{Control}

IP multicast is very new, and there are many problems that have not yet been solved. Since there is a lack of vendor support, most problems are being solved through a collective effort. Usually problems, concerns, questions, etc. are posted to mailing lists (e.g., mbone@ISI.EDU) where other MBONE users may help. Such lists are a source of information on conference scheduling or the latest tools. One problem is the lack of control on the MBONE. Other than peer pressure, nothing automatically manages the operation of the MBONE. The use of low $t t l$ values at the application level does help in keeping local multicast traffic from flooding the Internet. An additional problem is that IP multicast does not 
currently provide privacy; this is left for the user to provide. Plus, IP multicast sockets are UDP-based, which means that the MBONE only provides a best-effort service. Presently, most MBONE tools are best-effort. The user is responsible for any necessary reliability, privacy and control measures, as well as scheduling an event.

\subsubsection{Availability}

Currently, there are very few machines that are IP multicast capable. Mosedale[5] lists locations for obtaining software for reconfiguring some machines, information on connecting to the $\mathrm{MBONE}$, and information about obtaining and using MBONE tools. According to Mosedale, the following come with kernel support in place: Solaris (2.1+), BSD/386 (1.1+), DEC OSF/1 (2.0+), and IRIX (4.0+). For some systems that do not support IP multicast, software for reconfiguring the kernel exists. All of the machines used in this research had to be reconfigured. Also, one IP multicast-capable machine per subnet had to run the mrouted program to route multicast packets since routers in general are not capable of routing IP multicast packets.

\subsubsection{Address and Port assignments}

In this work, the address and port number were static. For testing purposes this was helpful because one would always know what address and port number to watch while using network debugging tools. For general use, this will cause problems especially if two users on the same network choose the same values.

A better way to choose these numbers is through random selection, but there will be clashes all too soon according to the birthday effect. To use McCanne and Jacobson's $s d[15]$ program, which greatly reduces the occurrence of an address/port clash, would be a better way of selecting these numbers. $S d$ randomly generates the numbers, and it avoids selecting those numbers that it hears in advertisements from other sites. However, $s d$ does not currently have an application interface. To use $s d$ with an application, one would need to enter the data as command line arguments, or the application would have to prompt the user for the information. According to Stephen $\mathrm{Casner}^{3}$, the authors of $s d$ plan to make a program interface. This would mean that a program would not have to generate its own address and port number or input the data manually. Also, although there may be no clashes between the host from which $s d$ was run, there possibly could be clashes for other hosts in the multicast group if those hosts are

\footnotetext{
${ }^{3}$ CASNER@ISI.EDU via email Wed., Sept. 14, 1994
} 
not within the same subnet. In this case, one may wish to test the address/port numbers for all hosts in a particular multicast group when using a WAN.

\subsubsection{Bandwidth and Routing}

Currently, the MBONE's bandwidth is only about 500kbps. As IP multicast and the multimedia applications that use it becomes more widely used, the need for more bandwidth will increase. Smarter multicast routing algorithms can prune back multicast traffic associated with inactive sessions and thus make better use of existing bandwidth. Researchers are already looking into making IP multicast routers more widely available which should decrease the number of hops and increase the available bandwidth.

If speed-up is the objective, reliable IP multicast over a WAN is not ready for production. Since IP multicast relies on specialized routers that are few in number compared to ordinary routers, an IP multicast packet incurs a large amount of overhead on a WAN. For instance, encapsulating a packet to be forwarded from one mrouter to another adds a small cost that unicast packets do not have. In addition, this cost is increased for each hop through the MBONE. In many cases, the IP multicast packet may have to travel hundreds of miles through the MBONE while a unicast packet with the same source and destination may travel only tens of miles. As well, for each hop, the probability of a packet being lost may increase.

\subsection{Future Work}

The MBONE's use is increasing rapidly. Internet users are discovering the uses or new uses for the MBONE. However, for it to grow, problems need to be solved and limitations overcome. To provide reliable IP multicast to the entire MBONE community, several things need to happen. An RFC for a reliable IP multicast protocol is needed such that reliability can be easily provided to MBONE applications and in order to standardize the method. More reliable IP multicast software and tools are needed before the MBONE can move into commercial use. A dynamic address and port server with which applications can interface is also needed. More IP multicast routers are needed in order to increase the bandwidth and decrease the number of hops through the MBONE making reliable IP multicast more competitive. Changes such as these would prepare IP multicast for production use.

For the current status and availability of IP multicast in PVM, the reader is invited to visit $h t t p: / / w w w . e p m . o r n l . g o v / p v m$. 


\section{References}

[1] A. S. Tanenbaum. Computer Networks Second Edition, chapter 1. Prentice Hall, 1989.

[2] C. Bormann, J. Ott, H.-C. Gehrcke, T. Kershat, and N. Seifert. MTP2: Towards Achieving the S.E.R.O Properties for Multicast Transport. In Presented at the ICCCN '94, San Francisco, September 1994.

[3] C. Huang, E. P. Kasten, and P. K. McKinley. Design and Implementation of Multicast Operations for ATM-Based High Performance Computing. In Proc. Supercomputing '94, Washington D.C., November 1994.

[4] D. Cheriton. VMTP: Versatile Message Transaction Protocol. Request for Comments 1045, Stanford University, 1988.

[5] D. Mosedale. Dan's Quick and Dirty Guide to Getting Connected to the MBONE. 1994. file://genome-ftp.stanford.edu/pub/mbone/mboneconnect.

[6] D. Waitzman, C. Partridge, and S. Deering. Distance Vector Multicast Routing Protocol. Request for Comments 1075, BBN STC, and Stanford University, 1988.

[7] G. A. Geist, A. Beguelin, J. Dongarra, W. Jiang, R. Manchek, and V. Sunderam. PVM: Parallel Virtual Machine a User's Guide and Tutorial for Networked Parallel Computing. The MIT Press, 1994.

[8] J. Mogul. Broadcasting Internet Datagrams. Request for Comments 919, Stanford University, 1984.

[9] K. Hall. The Implementation and Evaluation of Reliable IP Multicast. Master's thesis, University of Tennessee, Knoxville, 1994.

[10] K. Hwang. Advanced Computer Architecture: Parallelism, Scalability, Programmability, chapter 7. MIT Press and McGraw-Hill, Inc., 1993.

[11] K. Lebowitz and D. Mankins. Multi-network Broadcasting within the Internet. Request for Comments 947, BBN Laboratories, 1985.

[12] K. P. Birman and T. A. Joseph. On Communication Support for Fault Tolerant Process Groups. Request for Comments 992, Cornell University, 1986. 
[13] M. Ahamad and N. E. Belkeir. Using Multicast Communication for Dynamic Load Balancing in Local Area Networks. by personal communication.

[14] M. Kaashoek and A. S. Tanenbaum. Efficient Reliable Group Communication for Distributed Systems. (Submitted for publication 1994).

[15] M. Macedonia and D. Brutzman. MBONE, the Multicast BackbONE. IEEE Computer, January 1994. (draft article accepted for publication).

[16] M. Satyanarayanan, E. H. Siegel. Parallel Communication in a Large Distributed Environment. IEEE Transactions on Communications, 39(3):328348, March 1990.

[17] M. Satyanarayanan, J. J. Kistler, P. Kumar, M. E. Okasaki, E. H. Siegel, D. C. Steere. Coda: A Highly Available File System for a Distributed Workstation Environment. IEEE Transactions on Communications, 39(4):447-458, April 1990.

[18] R. Braudes and S. Zabele. Requirements for Multicast Protocols. Request for Comments 1458, TASC, May 1993.

[19] R. Manchek. Design and Implementation of PVM Version 3. Master's thesis, University of Tennessee, Knoxville, 1994.

[20] S. Armstrong, A. Freier, and K. Marzullo. Multicast Transport Protocol. Request for Comments 1301, Xerox, Apple, and Cornell University, 1992.

[21] S. Chang, D. Du, J. Hsieh, M. Lin, R. Tsang. Parallel Computing Over a Cluster of Workstations Interconnected via a Local ATM Network. Technical report, University of Minnesota Twin Cities, September 1994.

[22] S. Deering. Host Extensions for IP Multicasting. Request for Comments 1112, Stanford University, 1989.

[23] S. Pingali, D. Towsley, and J. Kurose. A Comparison of Sender-Initiated and Receiver-Initiated Reliable Multicast Protocols. ACM, SIGMETRICS 94, May 1994.

[24] T. Ballardie, P. Francis, and J. Crowcroft. Core Based Trees (CBT) An Architecture for Scalable Inter-Domain Multicast Routing. SIGCOMM '93, San Francisco, September 1993. 
[25] V. S. Sunderam, G. A. Geist, J. Dongarra, and R. Manchek. The PVM Concurrent Computing System: Evolution, Experiences, and Trends. Parallel Computing, 1992.

[26] W. T. Strayer, B. J. Dempsey, and A. C. Weaver. XTP: The Xpress Transfer Protocol, chapter 8. Addison-Wesley Publishing Company, Inc, 1992. 
ORNL/TM-13030

\section{INTERNAL DISTRIBUTION}

1. T. S. Darland

2. J. J. Dongarra

3-7. T. H. Dunigan

8. G. A. Geist

9. K. L. Kliewer

10. C. E. Oliver

11. R. T. Primm

12-16. S. A. Raby

17-21. M. R. Leuze
22-26. R. F. Sincovec

27. P. H. Worley

28. Central Research Library

29. ORNL Patent Office

30. K-25 Appl Tech Library

31. Y-12 Technical Library

32. Laboratory Records - RC

33-34. Laboratory Records Department

\section{EXTERNAL DISTRIBUTION}

35. Cleve Ashcraft, Boeing Computer Services, P.O. Box 24346, M/S 7L-21, Seattle, WA 98124-0346

36. Lawrence J. Baker, Exxon Production Research Company, P.O. Box 2189, Houston, TX 77252-2189

37. Clive Baillie, Physics Department, Campus Box 390, University of Colorado, Boulder, CO 80309

38. Jesse L. Barlow, Department of Computer Science, 220 Pond Laboratory, Pennsylvania State University, University Park, PA 16802-6106

39. Chris Bischof, Mathematics and Computer Science Division, Argonne National Laboratory, 9700 South Cass Avenue, Argonne, IL 60439

40. Ake Bjorck, Department of Mathematics, Linkoping University, S-581 83 Linkoping, Sweden

41. Roger W. Brockett, Wang Professor EE and CS, Div. of Applied Sciences, 29 Oxford St., Harvard University, Cambridge, MA 02138

42. James C. Browne, Department of Computer Science, University of Texas, Austin, TX 78712

43. Bill L. Buzbee, Scientific Computing Division, National Center for Atmospheric Research, P.O. Box 3000, Boulder, CO 80307

44. Donald A. Calahan, Department of Electrical and Computer Engineering, University of Michigan, Ann Arbor, MI 48109

45. Thomas A. Callcott, Director Science Alliance, 53 Turner House, University of Tennessee, Knoxville, TN 37996

46. Ian Cavers, Department of Computer Science, University of British Columbia, Vancouver, British Columbia V6T 1W5, Canada

47. Tony Chan, Department of Mathematics, University of California, Los Angeles, 405 Hilgard Avenue, Los Angeles, CA 90024 
48. Jagdish Chandra, Army Research Office, P.O. Box 12211, Research Triangle Park, NC 27709

49. Siddhartha Chatterjee, RIACS, MAIL STOP T045-1, NASA Ames Research Center, Moffett Field, CA 94035-1000

50. Eleanor Chu, Department of Mathematics and Statistics, University of Guelph, Guelph, Ontario, Canada N1G 2W1

51. Tom Coleman, Department of Computer Science, Cornell University, Ithaca, NY 14853

52. Paul Concus, Mathematics and Computing, Lawrence Berkeley Laboratory, Berkeley, CA 94720

53. Andy Conn, IBM T. J. Watson Research Center, P.O. Box 218, Yorktown Heights, NY 10598

54. John M. Conroy, Supercomputer Research Center, 17100 Science Drive, Bowie, MD 20715-4300

55. Jane K. Cullum, IBM T. J. Watson Research Center, P.O. Box 218, Yorktown Heights, NY 10598

56. George J. Davis, Department of Mathematics, Georgia State University, Atlanta, GA 30303

57. Tim A. Davis, Computer and Information Sciences Department, 301 CSE, University of Florida, Gainesville, FL 32611-2024

58. Larry Dowdy, Computer Science Department, Vanderbilt University, Nashville, TN 37235

59. Iain Duff, Numerical Analysis Group, Central Computing Department, Atlas Centre, Rutherford Appleton Laboratory, Didcot, Oxon OX11 0QX, England

60. Patricia Eberlein, Department of Computer Science, SUNY at Buffalo, Buffalo, NY 14260

61. Albert M. Erisman, Boeing Computer Services, Engineering Technology Applications, P.O. Box 24346, M/S 7L-20, Seattle, WA 98124-0346

62. Geoffrey C. Fox, Northeast Parallel Architectures Center, 111 College Place, Syracuse University, Syracuse, NY 13244-4100

63. Robert E. Funderlic, Department of Computer Science, North Carolina State University, Raleigh, NC 27650

64. Professor Dennis B. Gannon, Computer Science Department, Indiana University, Bloomington, IN 47401

65. David M. Gay, Bell Laboratories, 600 Mountain Avenue, Murray Hill, NJ 07974

66. C. William Gear, NEC Research Institute, 4 Independence Way, Princeton, NJ 08540

67. W. Morven Gentleman, Division of Electrical Engineering, National Research Council, Building M-50, Room 344, Montreal Road, Ottawa, Ontario, Canada K1A 0R8

68. J. Alan George, Vice President, Academic and Provost, Needles Hall, University of Waterloo, Waterloo, Ontario, Canada N2L 3G1 
69. John R. Gilbert, Xerox Palo Alto Research Center, 3333 Coyote Hill Road, Palo Alto, CA 94304

70. Gene H. Golub, Department of Computer Science, Stanford University, Stanford, CA 94305

71. Joseph F. Grcar, Division 8245, Sandia National Laboratories, Livermore, CA 94551-0969

72. John Gustafson, Ames Laboratory, Iowa State University, Ames, IA 50011

73. Michael T. Heath, 2304 Digital Computer Laboratory, University of Illinois, 1304 West Springfield A venue, Urbana, IL 61801-2987

74. Don E. Heller, Scalable Computing Laboratory, Ames Laboratory, US Dept. of Energy, Iowa State University, 327 Wilhelm Hall, Ames, Iowa 50011-3020

75. Dr. Dan Hitchock ER-31, MICS, Office of Energy Research, U. S. Department of Energy, Washington DC 20585

76. Robert E. Huddleston, Computation Department, Lawrence Livermore National Laboratory, P.O. Box 808, Livermore, CA 94550

77. Lennart Johnsson, 592 Philip G. Hoffman Hall, Dept. of Computer Science, The University of Houston, 4800 Calhoun Rd., Houston, TX 77204-3475

78. Harry Jordan, Department of Electrical and Computer Engineering, University of Colorado, Boulder, CO 80309

79. Malvyn H. Kalos, Cornell Theory Center, Engineering and Theory Center Bldg., Cornell University, Ithaca, NY 14853-3901

80. Hans Kaper, Mathematics and Computer Science Division, Argonne National Laboratory, 9700 South Cass Avenue, Bldg. 221, Argonne, IL 60439

81. Kenneth Kennedy, Department of Computer Science, Rice University, P.O. Box 1892, Houston, TX 77001

82. Dr. Tom Kitchens ER-31, MICS, Office of Energy Research, U. S. Department of Energy, Washington DC 20585

83. Richard Lau, Office of Naval Research, Code 1111MA, 800 Quincy Street, Boston, Tower 1, Arlington, VA 22217-5000

84. Alan J. Laub, Department of Electrical and Computer Engineering, University of California, Santa Barbara, CA 93106

85. Robert L. Launer, Army Research Office, P.O. Box 12211, Research Triangle Park, NC 27709

86. Charles Lawson, MS 301-490, Jet Propulsion Laboratory, 4800 Oak Grove Drive, Pasadena, CA 91109

87. Professor Peter Lax, Courant Institute for Mathematical Sciences, New York University, 251 Mercer Street, New York, NY 10012

88. John G. Lewis, Boeing Computer Services, P.O. Box 24346, M/S 7L-21, Seattle, WA 98124-0346

89. Franklin Luk, Electrical Engineering Department, Cornell University, Ithaca, NY 14853 
90. Paul C. Messina, Mail Code 158-79, California Institute of Technology, 1201 E. California Blvd., Pasadena, CA 91125

91. James McGraw, Lawrence Livermore National Laboratory, L-306, P.O. Box 808, Livermore, CA 94550

92. Dr. David Nelson, Director of Scientific Computing ER-30, Applied Mathematical Sciences, Office of Energy Research, U. S. Department of Energy, Washington DC 20585

93. Professor V. E. Oberacker, Department of Physics, Vanderbilt University, Box 1807 Station B, Nashville, TN 37235

94. Dianne P. O'Leary, Computer Science Department, University of Maryland, College Park, MD 20742

95. James M. Ortega, Department of Applied Mathematics, Thornton Hall, University of Virginia, Charlottesville, VA 22901

96. Charles F. Osgood, National Security Agency, Ft. George G. Meade, MD 20755

97. Roy P. Pargas, Department of Computer Science, Clemson University, Clemson, SC 29634-1906

98. Beresford N. Parlett, Department of Mathematics, University of California, Berkeley, CA 94720

99. Merrell Patrick, Department of Computer Science, Duke University, Durham, NC 27706

100. Robert J. Plemmons, Departments of Mathematics and Computer Science, Box 7311, Wake Forest University, Winston-Salem, NC 27109

101. James Pool, Caltech Concurrent Supercomputing Facility, California Institute of Technology, MS 158-79, Pasadena, CA 91125

102. Alex Pothen, Department of Computer Science, Pennsylvania State University, University Park, PA 16802

103. Professor Daniel A. Reed, Computer Science Department, University of Illinois, Urbana, IL 61801

104. John R. Rice, Computer Science Department, Purdue University, West Lafayette, IN 47907

105. Donald J. Rose, Department of Computer Science, Duke University, Durham, NC 27706

106. Joel Saltz, Dept. of Computer Science and Institute for Advanced Computer Studies, 4143 A. V. Williams Bldg., University of Maryland, College Park, MD 20742-3255

107. Martin H. Schultz, Department of Computer Science, Yale University, P.O. Box 2158 Yale Station, New Haven, CT 06520

108. David S. Scott, Intel Scientific Computers, 15201 N.W. Greenbrier Parkway, Beaverton, OR 97006

109. Kermit Sigmon, Department of Mathematics, University of Florida, Gainesville, FL 32611 
110. Horst Simon, NERSC Division, Lawrence Berkeley National Laboratory, Mail Stop $50 \mathrm{~A} / 5104$, University of California, Berkeley, CA 94720

111. Danny C. Sorensen, Department of Mathematical Sciences, Rice University, P. O. Box 1892, Houston, TX 77251

112. G. W. Stewart, Computer Science Department, University of Maryland, College Park, MD 20742

113. Paul N. Swartztrauber, National Center for Atmospheric Research, P.O. Box 3000, Boulder, CO 80307

114. Phuong Vu, Cray Research, Inc., 19607 Franz Rd., Houston, TX 77084

115. Robert Ward, Department of Computer Science, 107 Ayres Hall, University of Tennessee, Knoxville, TN 37996-1301

116. Andrew B. White, Computing Division, Los Alamos National Laboratory, P.O. Box 1663 MS-265, Los Alamos, NM 87545

117. David Young, University of Texas, Center for Numerical Analysis, RLM 13.150, Austin, TX 78731

118. Office of Assistant Manager for Energy Research and Development, U.S. Department of Energy, Oak Ridge Operations Office, P.O. Box 2001 Oak Ridge, TN 37831-6269

119-120. Office of Scientific \& Technical Information, P.O. Box 62, Oak Ridge, TN 37831 